\title{
Optimization of PID Parameters Based on Ant Colony Genetic Hybrid Algorithm
}

\author{
Gailian Zhang \\ School of Applied Technology, Xi'an International University, Xi'an, Shaanxi, PR. \\ China 710077
}

\begin{abstract}
Aiming at the disadvantage of slow convergence and low efficiency of genetic algorithm, ant colony algorithm is easy to fall into the local optimal solution. The optimization of PID parameters based on ant colony genetic hybrid algorithm is proposed. At last, in order to prove the effectiveness and feasibility of the algorithm, in the experiment simulation, the ant colony genetic algorithm The hybrid algorithm is compared with the traditional Z-N method and ant colony algorithm, and the conclusion is given at the end of the paper.
\end{abstract}

Keyword: PID control; ant colony algorithm; genetic algorithm; parameter optimization

\section{Introduction}

PID control as a earlier development of the control method, in the process control has been the most widely used, while PID parameters of self-tuning technology is also leaps and bounds, one is thanks to the rapid development of microelectronics technology led the PID parameter self-tuning technology, And the second is the development of model predictive control, which requires the PID controller to have good self-tuning ability. There are a lot of PID parameter optimization methods. Such as PID control method based on intelligent technology ${ }^{[1]}$, PID design method based on internal model control ${ }^{[2]}$, PID design method based on Smith predictor ${ }^{[3]}$, and ant colony optimization algorithm used in recent years ${ }^{[4]}$.

In this paper, the ant colony genetic hybrid algorithm is used to optimize the PID parameters. Secondly, the basic concept of ant colony algorithm is introduced. Then, a new algorithm combining ant colony algorithm and genetic algorithm is proposed to optimize the PID parameters. In order to prove the effectiveness of the algorithm, Effectiveness and feasibility of the ant colony algorithm. In the experiment simulation, the ant colony genetic hybrid algorithm is compared with the traditional Z-N method and the ant colony algorithm, and the conclusion is given at the end of the article.

2. General PID parameter tuning method

Today's industrial control, a variety of advanced control methods to be applied, but PID control is used in the production process or the main algorithm. Especially in the petrochemical industry, PID control of the control loop can reach $90 \%$. Regardless of the tedious field lines, the design of the main ideas are designed using the P I D control scheme, so that the system can effectively overcome the interference. The control operation relationship shown in equation (1) below 


$$
u(t)=K_{p}\left[e(t)+\frac{1}{T_{I}} \int_{0}^{t} e(t) d t+T_{D} \frac{d e(t)}{d t}\right]
$$

Formula: $e(t)=r(t)-y(t)$ the difference between the output signal and the reference value; $K_{p}$ Proportional gain; $T_{I}$ Integral time constant; $T_{D}$ Differential time constant; $K_{p} 、 T_{I} 、 T_{D}$ Three PID parameters.

When there is interference, given a signal change caused by bias, we want to use PID control system can be quickly returned to normal. However, if you want to achieve such a requirement, you need to have a set of suitable PID parameters before the design. A reasonable set of PID control parameters is required to get a set of tuning methods, the main PID tuning methods are as follows: (1) critical ratio Method, (2) decay curve method, (3) experience of try.

With the popularization of PLC and DCS, most of the self-tuning methods have been written into the software package on the system, these manufacturers own software package for the control of the project has been a huge help. The principle of the self-tuning software of the manufacturer is similar in general. It consists of three parts: the generation of process disturbance; the evaluation of disturbance response; and the calculation of controller parameters. These software greatly reduce the manual operation of the operator.

3. Ant colony algorithm

Ant colony algorithm ${ }^{[5]}$ is a typical intelligent optimization algorithm, the algorithm is very suitable for use with other algorithms, is mainly used in engineering optimization problems. Scientific and long-term experiments show that ants in the nature can get out of the current optimal path of a food to the cave in the invisible situation, and as the environment changes, if the previous path is no longer the current optimal, can continue Find the optimal path.

The ant colony algorithm is described by the traveling salesman optimization problem ${ }^{[6]}$. There are $\mathrm{n}$ cities A1, A2, A3 and so on. The distance between city Ai and $\mathrm{Aj}$ is denoted as $d_{\mathrm{ij}}$, The solution of such a path optimization problem is: In each of these cities, the total permutation and combination of each place leads to the shortest total path.Set the number of ants $\mathrm{m}, d_{\mathrm{ij}}$ Both $\mathrm{i}, \mathrm{j}$ the distance、 $\eta_{\mathrm{ij}}$ for(i,j)Of the visibility ; $\tau_{i j}$ for $(\mathrm{i}, \mathrm{j})$ Edge pheromone trajectory strength; $P_{\mathrm{ij}}^{\mathrm{k}}$ Is the transition probability of ants $\mathrm{k} ; \alpha$ As the relative importance of pheromones; $\beta$ and the relative importance of visibility。 allowed $_{k}=\left\{n-t a b u_{k}\right\}$ Indicates an ant $\mathrm{k}$ next to allow selection of the city。 $K$ in $t$, The probability of transition from one place $\mathrm{j}$ to another $P_{i j}^{k}(t)$ : 


$$
P_{i j}^{k}(t)=\left\{\begin{array}{l}
\frac{\left[\tau_{i j}(t)\right]^{\alpha} \cdot\left[\eta_{i j}(t)\right]^{\beta}}{\sum_{k \in \text { allowed }_{k}}\left[\tau_{i k}(t)\right]^{\alpha} \cdot\left[\eta_{i k}\right]^{\beta}} \quad j \in \text { allowed }_{\mathrm{k}} \\
0 \quad \text { otherwise }
\end{array}\right.
$$

When a loop is completed, the pheromone on the path that $\mathrm{k}$ ant walks is updated by formula (3).

$$
\Delta \tau_{i j}^{k}(t, t+n)=\left\{\begin{array}{l}
Q / L_{k} \text { if the Kth ant use edge }(i, j) \\
0 \quad \text { othersie }
\end{array}\right.
$$

Pheromone track updated to

$$
\begin{gathered}
\tau_{i, j}(t+n)=\rho_{1} \cdot \tau_{i j}(t)+\Delta \tau_{i j}(t, t+n) \\
\Delta \tau_{i j}(t, t+n)=\sum_{k=1}^{m} \Delta \tau_{i j}^{k}(t, t+n)
\end{gathered}
$$

$\rho$ Pheromone trajectory of the persistence.

The above formula shows that the change rule of the pheromone size in the path is more pheromone to the short path, so the positive feedback mechanism appears, like the enhanced learning algorithm, and the better solution can get more enhancement.

4 PID parameter optimization based on ant colony genetic hybrid algorithm

4.1 Ant algorithm optimization PID parameter basic idea

If you use the ant colony algorithm tuning PID parameters, need to be transformed into COP problem before solving P.

In the first step, we need to know the range of these parameters, so as to avoid too much solution to optimize the solution domain so that the convergence rate slows down. In this paper, the Z-N method is used firstly, $K_{p} 、 T_{I} 、 T_{D}$ and then a space is obtained based on the obtained parameters. This range is a feasible solution space, which reduces the searching range. The mathematical expression is as follows:

$$
\left\{\begin{array}{l}
(1-\lambda) K_{P} \leq K_{P}^{*} \leq(1+\varepsilon) K_{P} \\
(1-\lambda) T_{\mathrm{i}} \leq T_{\mathrm{i}}^{*} \leq(1+\varepsilon) T_{\mathrm{i}} \\
(1-\lambda) T_{\mathrm{d}} \leq T_{\mathrm{d}}^{*} \leq(1+\varepsilon) T_{\mathrm{d}}
\end{array}\right.
$$

Where $\mathrm{Z}$ is a random number chosen between zero and one, and then a feasible solution space for the parameters to be optimized is obtained, which is convenient for the ant colony algorithm to be optimized. When the size of the solution is close to the feasible solution space, we can expand the scope of the solution based on the solution, and then continue to search for the next round. After the feasible solution space is determined, the solution space will be discretized accordingly, so the ant colony algorithm will be easy to solve. $\lambda=0, \varepsilon=0.5$, divide the available solution space into 100 equal divisions, resulting in three discrete sets, each of which is a column. This 
PID parameter optimization problem will be converted into how the three sets which find three values, the combination of parameters, you can make the PID control to achieve optimal results.

Based on the ant colony optimization algorithm of PID control parameters can be simulated into, $K_{p} 、 T_{I} 、 T_{D}$ the three sets of the number as a collection of cities, ants starting at the starting point, through the set of all the cities, and finally to the end, and reach The criterion function index yields a shortest path. This problem can be seen as an ATSP problem, this will become a COP problem.

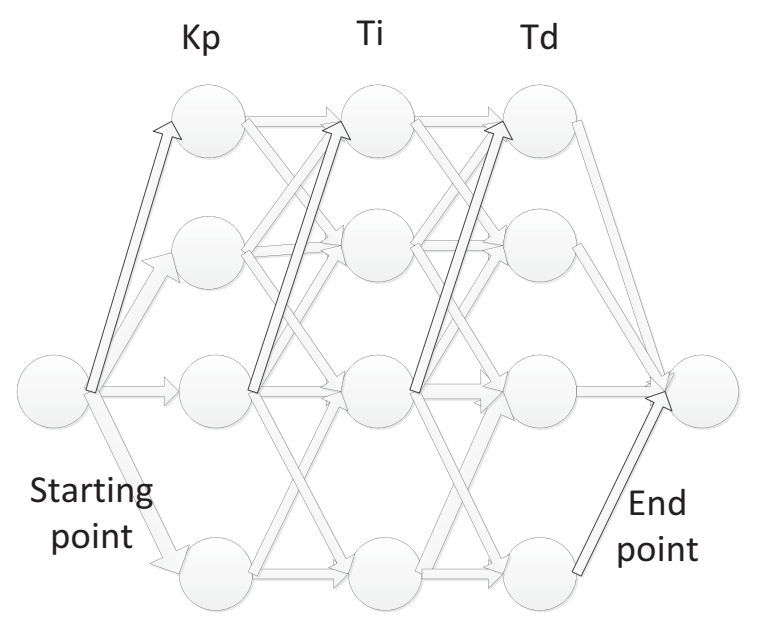

Figure 1 shows the optimization of PID control parameters based on ant colony algorithm

Which is not equivalent to the ATSP problem. The optimal solution of the ant colony algorithm is the optimal performance index of the control performance. The performance of the parameters of the merits of these parameters in the ant path on the node value shown on the pheromone is released in the ants through the nodes. The change of the pheromone size is no longer judged by the length of the path. The criterion function is used to change the pheromone size. The criterion function contains the information of all the nodes that the ant walks through and the current performance index of the system. A control system to measure, are from the stability, accuracy and speed of these three terms. The rise time of the system response represents the fastness, the shorter the rise time, the faster the response speed of the system. Of course, not only the fast response speed, this parameter will make the system control is too large, the system instability. Therefore, to obtain an optimal control performance, we need to obtain the following criterion function as the constraint condition of the system's control quantity, control error and rise time:

$$
J=\int_{0}^{\infty}\left(w_{1}|e(t)|+w_{2} u^{2}(t)\right) d t+w_{3} \cdot t_{u}
$$

In order to avoid overshoot, a penalty factor criterion function is added to modify the criterion function:

If ey $(t)<0$ 


$$
J=\int_{0}^{\infty}\left(w_{1}|e(t)|+w_{2} u^{2}(t)+w_{4}|e y(t)|\right) d t+w_{3} t_{u}
$$

$w_{4}$ Weight, and $w_{4} \geq w_{1}, e y(t)=y(t)-y(t-1), y(t)$ For the object to be pulled output

According to the above analysis, it can be concluded that the optimization of PID parameters by ant colony algorithm can be described as follows: In the first step, the feasible solution space is determined by formula (5), and then it is discretized. Initial time to initialize the ant to the starting point $S$, all the ants according to equation (2) to select the pseudo-random probability of the next path. The ant then passes through the corresponding nodes and releases the pheromone, and writes the nodes that have traveled to the memory list. When all the ants go from the starting point $\mathrm{S}$ to the end point $\mathrm{D}$, the value of the criterion function is obtained from the node order written in the memory list, and then the pheromone size is changed by Eq. (4). And then the next optimization is carried out. The final termination condition is set such that the criterion function $\mathrm{J}$ satisfies certain conditions, or iteratively determines the number of iterations.

4.2 The basic idea of genetic algorithm

Genetic algorithm ${ }^{[7]}$ is a global optimization search method, the basic idea of this algorithm is based on the survival of the competition, the survival of the fittest survival of the fittest, genetic algorithm optimization goal is a population of all individuals, the population for a series The genetic algorithm is used to change the fitness value of the individual fitness, and then it loops until a global heuristic random search algorithm is found for the optimal individual.

In general, the initial population is randomly generated, and then the fitness function $\mathrm{f}(\mathrm{x})$ ( $\mathrm{x}$ represents the individual) to evaluate the merits of the individual. The basic operation of genetic algorithm for the three: genetic, crossover and mutation.

Genetic algorithm has the advantage of global capacity, the disadvantage is that convergence is slow, low efficiency. The advantage of the ant colony algorithm is that it is based on positive feedback mechanism, but the disadvantage is very easy to fall into the local optimal solution. Therefore, this paper combines two algorithms, and proposes an ant colony genetic hybrid algorithm to optimize the PID parameters.

4.3 Ant Colony Genetic Hybrid Algorithm PID parameter optimization steps

The optimization of PID parameters of ant colony genetic hybrid algorithm is as follows:

A) From the traditional Z-N method to obtain the PID parameter values, $K_{P, Z-N}$ 、

$K_{i, Z-N}$ and $K_{\mathrm{d}, Z-N}$

B) Set $\mathrm{m}$ ant, ant $\mathrm{k}(\mathrm{k}=1 \sim \mathrm{m})$ has 15 stored ants passing node's ordinate value and passing path attribute.

C) Algorithm initialization. Let $\mathrm{t}=0,=0$, assign $\mathrm{NCmax}$ and initial moment $\tau\left(x_{\mathrm{i}}, y_{i, j}, 0\right)$ Assignment $(\mathrm{i}=1 \sim 15, \mathrm{j}=0 \sim 9) . \Delta \tau\left(x_{\mathrm{i}}, y_{i, j}\right)=0$ put all ants 
at the starting point.

D) $i=1$, if, from (2) the ant to all nodes on the line segment transfer probability; otherwise the next node with roulette, and select the value of the node tabu table.

E) When ants passes through a node, the local pheromone size is changed by Eq. (4).

F) $\mathrm{i}=\mathrm{i}+1$, if $\mathrm{i} \leq 15$, go to step c); otherwise, perform $\mathrm{g}$ ).

G) According to the array and calculate the corresponding PID parameters of the path;

$K_{\mathrm{p}}^{\mathrm{k}}, K_{\mathrm{i}}^{\mathrm{k}}, K_{\mathrm{d}}^{\mathrm{k}}$ and then simulate the system performance indicators, steady-state adjustment error and overshoot; according to equation (7) to calculate the corresponding function of the ant $\mathrm{k}$; record the current round of the cycle Optimal path and optimal performance index, and will be deposited in the.

H) $\mathrm{t} \leftarrow \mathrm{t}+15 ; N_{C} \leftarrow N_{C}+1$;

I) Generation of new individuals using cross-policy.

J) Take the mutation strategy and recalculate the parameters. If the calculated performance index is close to the objective function $\mathrm{J}$, the mutation can be saved. Otherwise, the mutation will be canceled.

K) If, and if all ants do not converge to the same path, then all ants need to be placed at the starting point, go to step d); otherwise end the loop to arrive at the optimal path and optimal PID parameters.

5. Experimental analysis

The following is a simulation of the actual object to analyze the effectiveness of the proposed algorithm, the actual object to be selected more complex objects, this can clearly reflect the advantages of the algorithm, the impact of hydraulic rotary drilling rig electrohydraulic servo system for the typical non-linear, A large lagged control object whose transfer function can be described as: Using the unit step function as the input signal for optimization, and using the algorithm proposed in this paper and other algorithms for comparison.

Before the simulation, the parameters of the algorithm need to be selected, the parameters are as follows: $\mathrm{Q}=1, \mathrm{p}=0.7, \quad \mathrm{a}=1$, the number of ants is 40 , and the number of iterations is 150 . PID control system parameters range: $\mathrm{Kp}$ is[0.00001 20], and the value of $\mathrm{Ki}$ and $\mathrm{Kd}$ is $[0.000012]$. In order to analyze the performance of the algorithm proposed in this paper, the ant colony genetic hybrid algorithm is compared with the $\mathrm{ZN}$ method and the ACO algorithm respectively. Fig. 2 is the comparison chart of the algorithm. Table 1 shows the optimized parameters of $\mathrm{ZN}$, ACO and GA-ACO. Out of the data comparison. 


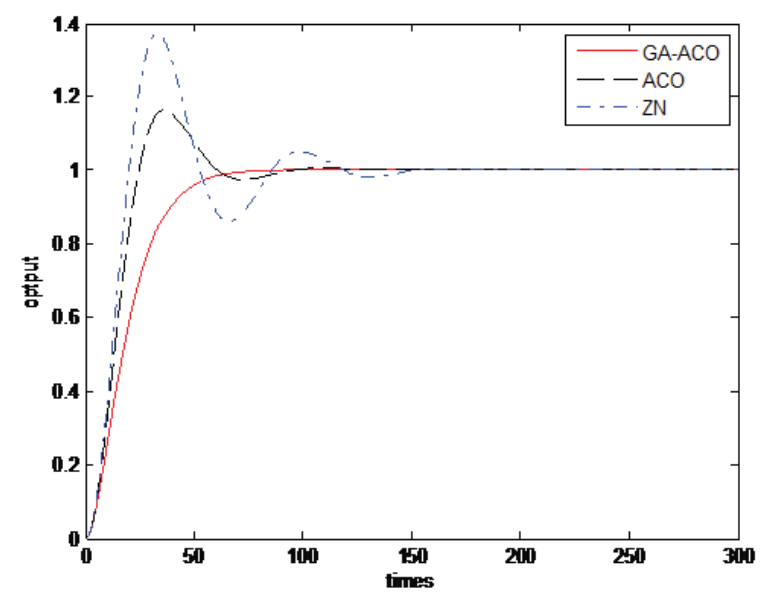

Figure 2 Comparison of the algorithm

Table 1 three kinds of methods to optimize the PID parameters and performance indicators

\begin{tabular}{|l|l|l|l|l|l|l|}
\hline algorithm & $\mathrm{Kp}$ & $\mathrm{Ki}$ & $\mathrm{Kd}$ & ts & ess & ct \\
\hline Z-N & 3.56 & 1.8 & 0.7 & 32.85 & $7.258 \mathrm{e}-8$ & 30.5874 \\
\hline ACO & 8.56 & 1.7685 & 1.5248 & 14.62 & $3.968 \mathrm{e}-8$ & 16.2598 \\
\hline GA-ACO & 7.2002 & 0.0154 & 0.5874 & 10.26 & 0.1472 & 0.1472 \\
\hline
\end{tabular}

As can be seen from the comparison data in Table 1, the PID parameters optimized by ACS-GA presented in this paper are the best.

6. Concluding remarks

Genetic algorithm has the advantage of global capacity, the disadvantage is that convergence is slow, low efficiency. The advantage of the ant colony algorithm is that it is based on positive feedback mechanism, but the disadvantage is very easy to fall into the local optimal solution. Therefore, this paper combines two algorithms, and proposes an ant colony genetic hybrid algorithm to optimize the PID parameters. The basic idea is to convert the PID parameter optimization problem into the COP problem, and then use the ant colony algorithm to optimize, and then use the genetic algorithm to get the solution to be further optimized, and finally get the optimal PID parameter. Experimental results show that the proposed algorithm not only avoids stagnation, but also improves global convergence, low parameter value sensitivity and greatly improves the solution efficiency.

\section{Acknowledgements}

This work was financially supported by Shaanxi Provincial Education Commission (ProgramNo.16JK2174)

\section{Author Introduction}

About The Author: Gailian Zhang (1978.11 ), Female, Master, Native place:Baotou, Inner Mongolia, college lecturer of School of Applied Technology in Xi'an 
International University. Research direction: Intelligent control, Electrical control technology and Application of PLC technology.

\section{references}

[1] Liu JK. Advanced PID control and its Matlab simulation [M]. Electronic Industry Press, 2003.

[2] Rotstein GE, Lewin DR.Simple PI and PID Type Controllers for Unstable Systems [J]. Industrial Engineering and Chemical Research, 1991, 30: 1864-1969.

[3] Cao Shunan, Xie Xuejun, Liu Guanglin. PID adaptive control based on Smith predictor and its application [J]. Chemical Industry and Automation, 2004, 31 (1): 28-83.

[4] Duan Haibin, Wang Daobo.PID parameter optimization based on ant colony algorithm [J] .Journal of Wuhan University: Engineering Science, 2004, 37 (5): 97

[5] WANG P, KWOK D. P. Optimal design of PID process controllers based on genetic algorithms [J] .Control Engineering Practice, 1994,2 (4): 641-648.

[6] Li Shiyong. Ant colony algorithm and its application [M]. Harbin: Harbin Institute of Technology, 2004: 22-104.

[7] Zhang Xianle, Jiang Hong. PID Parameter Optimization Based on Improved GAAA Algorithm [J] .Computer Measurement \& Control, 2006,14 (11): 1545-1547. 